\title{
Forced Oscillations of Transonic Channel and Inlet Flows with Shock Waves
}

\author{
A. F. Messiter* and T. C. Adamson Jr. $\dagger$ \\ The University of Michigan, Ann Arbor, Michigan
}

\begin{abstract}
In transonic channel or inlet flow, large-amplitude motions of a shock wave can be caused by small-amplitude oscillations of the walls or the back pressure. Previous work, which included only the latter effect, has been extended, and both types of impressed disturbances are considered here. Asymptotic solutions are obtained for the velocity and pressure distributions throughout a two-dimensional channel, with emphasis on flows which accelerate through sonic speed at the channel throat and with special attention given to small regions near the channel entrance and throat and near the shock wave. Solutions for the shock-wave velocity are derived, and for sufficiently strong disturbances it is shown how the shock wave moves rapidly upstream through the subsonic flow ahead of the throat. The details of the problem formulation and solution depend on the relative orders of magnitude of the nondimensional amplitude and frequency of the impressed oscillations; one case is considered in detail, and two others are discussed more briefly. Numerical results are shown for illustrative examples.
\end{abstract}

\section{Introduction}

$\mathbf{U}$ NSTEADY two-dimensional transonic channel flows have been studied in various limiting cases ${ }^{1-5}$ when perturbations are caused by small prescribed time-dependent pressure disturbances at some location downstream of a channel throat. In Refs. 1 and 2, for symmetric and asymmetric channels, respectively, the amplitude and frequency of the pressure disturbances were such that a shock wave located downstream of the throat experienced only small-amplitude oscillations. In subsequent work,,$^{3,4}$ large-amplitude shockwave motion caused by a somewhat slower pressure variation was examined; numerical results showed two possible configurations for the integral curves representing the motion, each of which included the possibility of the shock wave moving upstream through the throat and reappearing at a later time. Still another case ${ }^{5}$ of large-amplitude shock-wave motion arises if the amplitude, rather than the period, of the pressure disturbances is increased. The solutions for these various channel flows are also applicable to two-dimensional transonic flow between the blades of a cascade, as shown for steady flow in Ref. 6.

Similar types of flow occur if the unsteadiness is caused by motion of the channel walls. This paper, a shortened version of Ref. 7, describes the motions caused by a combination of the two types of disturbances; several minor errors in Ref. 7 have been corrected, and the notation has been changed slightly. The solutions of Ref. 3 are extended here to show both the similarities and the differences in the flow perturbations resulting from wall oscillations and oscillations in back pressure. Extensions of the other two cases mentioned above, $, 1,2,5$ corresponding to somewhat higher frequencies, are discussed more briefly. For all cases, since changes in crosssectional area are assumed small, in most of the channel the first approximation describes a one-dimensional flow, and higher approximations then can be derived rather easily. This perturbation scheme fails, however, in thin regions near the throat, just downstream of the shock wave, and at the channel

Received June 28, 1983; revision received Dec. 7, 1983. Copyright (C) 1984 by A. F. Messiter. Published by the American Institute of Aeronautics and Astronautics with permission.

*Professor, Department of Aerospace Engineering. Associate Fellow AIAA.

$\dagger$ Professor and Chairman, Department of Aerospace Engineering. Fellow AIAA. entrance and exit. In these regions the flow is twodimensional in a first approximation, but simplifications occur because only the local geometry is important, and again analytical solutions can be obtained. These are "inner" solutions which are matched asymptotically with the channel solution, or with a solution for the flow outside the channel. The extensions of the earlier solutions given here include detailed descriptions of the entrance and throat regions of a channel or an inlet. The solution in the entrance region is obtained by a procedure similar to that used in Ref. 6 . The region near the shock wave is discussed in Refs. 1 and 8 . Solutions for the shock-wave motion are also obtained when the shock wave is downstream of, close to, or upstream of the throat, and numerical examples are shown.

Since both means of introducing flow disturbances, through wall oscillations or oscillations in downstream pressure, are retained here, the solutions may have applications to the flow in inlets, to the flow between compressor blades in turbojet engines, or to the diffusers of ramjet engines, as long as the flow is transonic. Wall oscillations may be associated either with blade flutter or with the displacement effect of an unsteady turbulent boundary layer or separation bubble. Oscillations in downstream pressure may be caused by combustion instabilities, oscillating blade wakes, etc., downstream of the channel in question. The possibility of flutter has been discussed, e.g., in Ref. 9. Interactions between a core flow and regions of unsteady separated flow have been studied experimentally (e.g., Refs 10 and 11) and self-induced oscillations have been observed. Analytical studies ${ }^{12,13}$ have coupled the solutions of Refs. 8 and 3 with boundary-layer solutions; a solution describing interaction of the boundary layer and the shock wave was included in Ref. 13. A recent numerical study ${ }^{14}$ has had some success in predicting self-sustained oscillations with large regions of separated flow. Finally, in a ramjet engine, combustion instabilities might propagate upstream and cause oscillations in pressure ${ }^{15}$ and thus in the position of the shock wave; in some cases diffuser 'buzz"' could result.

\section{Asymptotic Problem Formulations}

A thin-walled duct is placed in a uniform steady flow at high subsonic Mach number, as in Fig. 1. The walls might also be regarded as blades in an unstaggered cascade; the exterior surfaces need not be plane. Rectangular coordinates $x$ and $y$, nondimensional with the half-width $L$, are measured along 


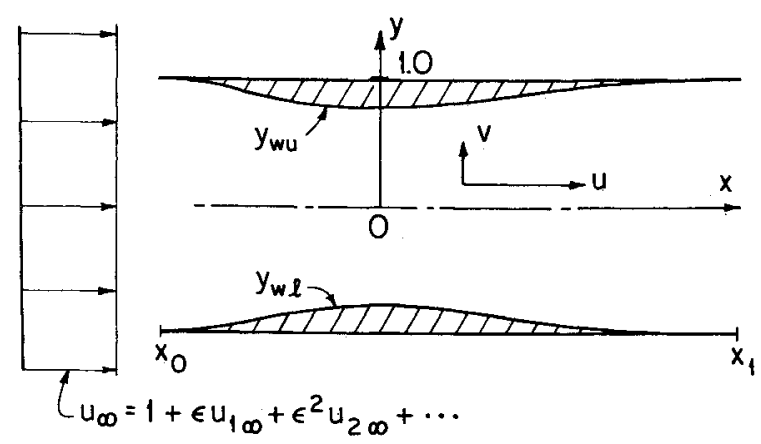

Fig. 1 Coordinate system and notation.

and normal to the direction of the undisturbed flow, with the origin on the centerline at the location of the minimum crosssectional area. The duct extends from $x=x_{0}<0$ to $x=x_{1}>0$; leading and trailing edges are shown as cusped, but might instead be wedge-shaped or rounded. The time $t$ has been made nondimensional with a characteristic time, say, $\omega^{-1}$, of the impressed oscillations; for simple harmonic oscillations, $\omega$ is the angular frequency. A nondimensional characteristic time is $\tau=\left(\omega L / a^{*}\right)^{-1}$, where $a^{*}$ is the critical sound speed. In the cases studied here, $\tau^{-1} \ll 1$; i.e., the reduced frequency $\omega L / a^{*}$ is small. by

The inner surfaces of the upper and lower walls are defined

$$
\begin{aligned}
& y_{w u}=1-\epsilon^{2} f_{u}(x)+\alpha G_{u}(x, t) \\
& y_{w \ell}=-1+\epsilon^{2} f_{\ell}(\mathrm{x})-\alpha G_{\ell}(x, t)
\end{aligned}
$$

where $\epsilon^{2} \ll 1$ and $\alpha \ll 1$. Thus $\epsilon^{2}$ is a small parameter which measures the wall (or blade) thickness ratio or the ratio of the duct half-width to a typical wall radius of curvature, and $\alpha$ is proportional to the nondimensional amplitude of the wall oscillations. For rigid walls the functions $G_{u}$ and $G_{\ell}$ would describe combined pitching and plunging motions with arbitrary phase relationships.

Velocity components $u$ and $v$, nondimensional with $a^{*}$, are in the $x$ and $y$ directions, respectively. The Mach number and the nondimensional velocity in the undisturbed flow are $M_{\infty}$ and $u_{\infty}$, respectively; each has a value slightly smaller than one. The discussion of shock-wave oscillations downstream of the throat, however, also remains correct if $M_{\infty}$ and $u_{\infty}$ are slightly larger than one. The pressure $p$, density $\rho$, temperature $T$, and sound speed $a$ have been made nondimensional with the corresponding critical values in the undisturbed flow. A perfect gas is assumed, with a constant ratio of specific heats $\gamma$.

Four small parameters now have been introduced: $\epsilon, \alpha$, $\tau^{-1}$, and $1-M_{\infty}^{2}$ (or $1-M_{\infty}$, or $1-u_{\infty}$ ). Asymptotic solutions are sought as these parameters tend to zero. The nature of the solution obtained, and thus the nature of the flowfield described, depends on the relative sizes of the parameters as the limit is taken. In all cases to be considered, the choice $1-M_{\infty}^{2}=\mathcal{O}(\epsilon)$ is made; velocity perturbations are then found to be the same order as $1-u_{\infty}$. The minimum Mach number for choking can be expressed by a series expansion in $\epsilon$. In order that this flow condition may be included as one possible example, $u_{\infty}$ and $M_{\infty}$ are expanded as a power series in $\epsilon$. The appropriate forms are

$$
u_{\infty}=I+\epsilon u_{I \infty}+\epsilon^{2} u_{2 \infty}+\ldots, \quad I-M_{\infty}^{2}=K \epsilon+\mathcal{O}\left(\epsilon^{2}\right)
$$

where $K=-(\gamma+1) u_{1 \infty}$ since $M_{\infty}=u_{\infty} / a_{\infty}$ and the nondimensional undisturbed sound speed $a_{\infty}$ is found from $a_{\infty}^{2} /(\gamma-1)+1 / 2 u_{\infty}^{2}=1 / 2(\gamma+1) /(\gamma-1)$. Three possibilities for $\alpha$ and $\tau^{-1}$ are considered; in each case, downstream pressure fluctuations $\Delta p$ are also introduced, with the same charac- teristic time as that for the wall oscillations, and with amplitude such that the effects are of the same order as the effects of the wall oscillations. The three cases are:

$$
\begin{aligned}
\text { 1) } \tau^{-1}=\mathcal{O}\left(\epsilon^{2}\right), \alpha=\mathcal{O}\left(\epsilon^{3}\right), \Delta p=\mathcal{O}\left(\epsilon^{2}\right) \\
\text { 2) } \tau^{-1}=\mathcal{O}(\epsilon), \alpha=\mathcal{O}\left(\epsilon^{3}\right), \Delta p=\mathcal{O}\left(\epsilon^{2}\right) \\
\text { 3) } \tau^{-1}=\mathcal{O}(\epsilon), \alpha=\mathcal{O}\left(\epsilon^{2}\right), \Delta p=\mathcal{O}(\epsilon)
\end{aligned}
$$

The steady-state downstream pressure at $x=x_{1}$ is assumed to lie in the range for which the flow is choked and a shock wave is present at some location downstream of the throat. Since a weak pressure disturbance travels upstream at a speed $|u-a|=\vartheta(\epsilon)$, the effects of a downstream disturbance can be considered to reach the shock wave instantaneously if $\tau^{-1}=\mathcal{O}\left(\epsilon^{2}\right)$, but will be delayed by a time comparable with the characteristic time of the disturbance if $\tau^{-1}=\mathcal{O}(\epsilon)$. In the first and third cases mentioned, the shock wave displacement is found to be $\Delta x_{s}=\mathcal{O}(1)$, whereas in the second case $\Delta x_{s}=\mathcal{O}(\epsilon)$. The first case is discussed in detail, and the others are sketched more briefly.

In all cases the shock wave is weak and nearly normal, so that the vorticity in the flow downstream of the shock wave is extremely small. To the order of magnitude considered here, a velocity potential exists, defined by $q=u i+v j=i+\nabla \phi$, or

$$
u=1+\phi_{x}, \quad v=\phi_{y}
$$

For irrotational isentropic flow, the integral of the momentum equation is

$$
\frac{1}{\tau} \phi_{t}+\frac{1}{2} q^{2}+\frac{a^{2}}{\gamma-l}=F(t)
$$

where $q^{2}=u^{2}+v^{2}$. The total enthalpy $H$, nondimensional with $a^{* 2}$, is defined by $H=a^{2} /(\gamma-1)+1 / 2 q^{2}$. If $\phi \rightarrow 0$ upstream, $F(t)$ is equal to the undisturbed value $H_{\infty}=1 / 2(\gamma+1) /(\gamma-1)$ in the flow ahead of the shock wave. Downstream of the shock wave, however, $F(t)$ is determined by the details of the shock-wave motion. The differential equation satisfied by $\phi$ is

$$
a^{2} \nabla^{2} \phi-q \cdot \nabla \frac{q^{2}}{2}-\frac{1}{\tau^{2}} \phi_{t t}+\frac{1}{\tau} F^{\prime}(t)-\frac{2}{\tau} q \cdot q_{t}=0
$$

Since $a^{2}=1+1 / 2(\gamma-1)\left(1-q^{2}\right)+(\gamma-1)\left(H-H_{\infty}\right)$, the potential equation (5) becomes

$$
\begin{aligned}
\phi_{y y} & =(\gamma+1) \phi_{x} \phi_{x x}+2\left(1+\phi_{x}\right) \tau^{-1} \phi_{x t}+\tau^{-2} \phi_{t t} \\
- & \tau^{-1} F^{\prime}(t)+(\gamma-1) \phi_{x} \phi_{y y}+1 / 2(\gamma+l) \phi_{x}^{2} \phi_{x x} \\
& -(\gamma-1)\left(H-H_{\infty}\right)\left(\phi_{x x}+\phi_{y y}\right)+\ldots
\end{aligned}
$$

All omitted terms are of higher order than terms retained in the following derivations, as can be verified afterward. The rates of change of $H$ and $p / \rho^{\gamma}$ along particle paths are

$$
\frac{\mathrm{D} H}{\mathrm{D} t}=\frac{l}{\gamma \rho} \frac{\partial p}{\partial t}, \quad \frac{\mathrm{D}\left(p / \rho^{\gamma}\right)}{\mathrm{D} t}=0
$$

The equation of state is $p=\rho T$ and the sound speed is found from $a^{2}=T$.

The shock-wave shape can be expressed in the form

$$
S(x, y, t)=0=x-x_{s}(y, t)
$$


A point which moves with velocity $c$ and remains on the shock wave is described by

$$
\tau^{-I} \frac{\mathrm{D} S}{\mathrm{D} t}=0=\tau^{-I} S_{t}+c \cdot \nabla S
$$

at $S=0$. The normal component $c_{n}$ and the normal component $q_{n}$ of the gas velocity at the shock wave are

$$
c_{n}=-\tau^{-1} S_{t} /|\nabla S|, \quad q_{n}=q \cdot \nabla S /|\nabla S|
$$

Subscripts $u$ and $d$ will be used to denote values of $q_{n}, u, H$, etc., immediately upstream and downstream of the shock wave, respectively. Since $\left|\partial x_{s} / \partial y\right|$ is sufficiently small, as can be verified later, $q_{n} \approx u$ and $c_{n} \approx \tau^{-1} \partial x_{s} / \partial t$. In a coordinate system fixed with respect to the shock wave, the total enthalpy is unchanged across the shock. Changing to the original coordinates then gives the jump in $H$ as

$$
H_{d}-H_{u}=-c_{n}\left(q_{n u}-q_{n d}\right) \approx-u_{s}\left(u_{u}-u_{d}\right)
$$

where $u_{s}=\tau^{-I} \partial x_{s} / \partial t$. The Prandtl relation, correct in coordinates fixed on the shock, becomes approximately

$$
\left(u_{u}-u_{s}\right)\left(u_{d}-u_{s}\right) \approx 2(\gamma-I) /(\gamma+l)\left[H_{u}-u_{s} u_{u}+1 / 2 u_{s}^{2}\right]
$$

The boundary condition specifying zero flow through the walls is

$$
v_{w}=\frac{1}{\tau} \frac{\partial y_{w}}{\partial t}+u_{w} \frac{\partial y_{w}}{\partial x}
$$

where $u_{w}$ and $v_{w}$ are the values of $u$ and $v$ at $y=y_{w}$, and the subscript refers to either the upper or the lower wall.

\section{Solutions for "Slow" Oscillations}

In the first case to be considered, the small parameters are specified by $\tau^{-1}=k \epsilon^{2}$ and $\alpha=\epsilon^{3}$; then $k$ is defined by $k=\epsilon^{-2}\left(\omega L / a^{*}\right)$. For example, a simple harmonic rigid pitching motion with symmetric wall deflections would be described by setting $G_{u}=G_{\ell}=\beta_{0}\left(x-x_{c}\right) e^{i t}$, where $\beta_{0}=$ const and the pitch axis is at $x=x_{c}$. While these oscillations might be called "slow," because the reduced frequency $\tau^{-1}=\omega L / a^{*}$ is a small number, the frequency $f=a^{*} \tau^{-1} /(2 \pi L)$ may still seem large when measured in $\mathrm{Hz}$. For example, if $a^{*}=330$ $\mathrm{m} / \mathrm{s}, L=0.03 \mathrm{~m}$, and $\tau^{-1}=0.04$, then $f=70 \mathrm{~Hz}$.

\section{Channel-Flow Solutions}

For most of the channel, the potential can be expanded in the form

$$
\phi=\epsilon \phi_{1}+\epsilon^{2} \phi_{2}+\epsilon^{3} \phi_{3}+\ldots
$$

Similar expansions can be written for $u-1$ and $v$, with $u_{I}=\phi_{I x}, v_{l}=\phi_{I y}$, etc. Retaining the largest terms in Eqs. (6) and (13), one finds simply

$$
\phi_{l y y}=0, \quad \phi_{I y}(x, \pm 1, t)=0
$$

It follows that $\phi_{I}=\phi_{I}(x, t)$. The next terms in Eq. (6) then give

$$
\phi_{2 y y}=(\gamma+1) \phi_{I x} \phi_{I x x}
$$

$\phi_{3 y y}=\left[(\gamma+1) \phi_{1 x} \phi_{2 x}+2 k \phi_{1 t}+1 / 3\left[(\gamma-1 / 2)(\gamma+1) \phi_{1 x}^{3}\right]_{x}\right.$ and the expansion of Eq. (13) yields

$$
\begin{array}{cl}
\phi_{2 y}(x, 1, t)=-f_{u}^{\prime}, & \phi_{2 y}(x,-1, t)=f_{\ell}^{\prime} \\
\phi_{3 y}(x, l, t)=G_{u x}-u_{1} f_{u}^{\prime}, & \phi_{3 y}(x,-1, t)=-G_{\ell x}+u_{l} f_{\ell}^{\prime}
\end{array}
$$

Integration of Eq. (16a) and substitution into Eq. (17a) then leads to a differential equation for $\phi_{l x}$ which is easily integrated to give

$$
\phi_{l x}= \pm\left[2\left(c_{l}-f\right) /(\gamma+1)\right]^{1 / 2}, \quad c_{l}=f(0)
$$

where $f=1 / 2\left(f_{u}+f_{\ell}\right)$; the upper and lower signs refer to supersonic and subsonic flow, respectively. If the flow everywhere in the duct were subsonic, the integration constant $c_{I}$ would be determined by conditions at the exit, whereas $c_{I}$ would be determined by entrance conditions if the entrance flow were supersonic. In the present case a subsonic flow is accelerated to sonic speed at the throat $x=0$, and $c_{1}$ is chosen so that $\phi_{1 x}=0$ at $x=0$. Thus $\phi_{1}$ is a function only of $x$.

The solution for $\phi_{2}$ has the form

$$
\phi_{2}=-1 / 2 f^{\prime} y^{2}+g y+h_{2}(x, t)
$$

where $g=1 / 2\left(f_{\ell}^{\prime}-f_{u}^{\prime}\right)$. Equation (16b) can be integrated once to give $\phi_{3 y}$, and substitution in Eq. (17b) leads to a differential equation for $h_{2 x}$ which is easily integrated to give

$$
h_{2 x}=\frac{1}{6}\left[f^{\prime \prime}-(2 \gamma-3) \phi_{I x}^{2}\right]+\frac{c_{2}(t)+G}{(\gamma+l) \phi_{I x}}
$$

where $G=1 / 2\left(G_{u}+G_{\ell}\right)$ and now $\phi_{I x}=\mathrm{d} \phi_{l} / \mathrm{d} x$. It will be seen below, from the shock-wave relations, that $c_{2}(t)$ is discontinuous at the shock wave. For choked flow, the upstream value $c_{2 u}(t)$ is found from the requirement that $\phi_{2 x}$ be finite at $x=0$ :

$$
c_{2 u}(t)+G(0, t)=0
$$

The downstream value $c_{2 d}(t)$ can be determined from a prescribed pressure variation at the exit $x=x_{1}$. Using $p \approx \rho^{\gamma}$, $p=\rho T$, and $a^{2}=T$, one finds using Eq. (5) that

$$
p=I+\epsilon p_{1}+\epsilon^{2} p_{2}+\ldots=1-\epsilon \gamma u_{1}-\epsilon^{2} \gamma u_{2}+\ldots
$$

If, for example, $f=f^{\prime}=f^{\prime \prime}=0$ at $x=x_{l}$, then at $x=x_{1}$

$$
-\frac{l}{\gamma} p_{2}=-\frac{l}{6}(2 \gamma-3) u_{I}^{2}\left(x_{I}\right)+\frac{c_{2 d}(t)+G\left(x_{1}, t\right)}{(\gamma+1) u_{I}\left(x_{l}\right)}
$$

and specification of $p_{2}$ as a function of $t$ at $x=x_{1}$ determines $c_{2 d}(t)$. The function of time appearing in Eq. (23) can be rewritten in the form

$$
c_{2 d}(t)+G\left(x_{1}, t\right)=c_{2 d}^{(s)}+G_{d}(t)
$$

where $c_{2 d}^{(s)}$ is the steady-state value ${ }^{8}$ of $c_{2 d}(t)$ which determines the steady-state shock-wave position, and $G_{d}(t)$ is proportional to the fluctuating term in the downstream pressure. Then $c_{2 d}(t)$ is defined by Eq. (24).

\section{Entrance Solution}

At the entrance of the channel or inlet, the nonuniform velocity found from Eq. (14) cannot be matched directly with the uniform upstream velocity, Eq. (2). Thus there is a need for an "entrance" or "leading-edge" solution, as was demonstrated in Ref. 6 for steady transonic flow through a 
cascade. In a thin vertical region containing the leading edges, the solution can contain enough information to describe the needed flow adjustment only if the terms $(\gamma+1) \phi_{x} \phi_{x x}$ and $\phi_{y y}$ are of the same order of magnitude there. If the upstream Mach number $M_{\infty}$ is assumed to be only slightly above the minimum value for choked flow, the term of order $\epsilon$ in the velocity remains constant near the entrance. The values $u_{l \infty}$ and $\phi_{I x}\left(x_{0}\right)$ are then the same. Since $f\left(x_{0}\right)=0$,

$$
u_{1 \infty}=-\left(\frac{2 c_{l}}{\gamma+1}\right)^{1 / 2}=-\left(\frac{2 f(0)}{\gamma+1}\right)^{1 / 2}
$$

For simplicity, the duct walls are assumed to have straight exterior surfaces, as in Fig. 1. If the leading edges are cusped, again as in Fig. 1, $\phi_{2 y}=\mathcal{O}\left(x-x_{0}\right)$ as $x \rightarrow x_{0}$. It follows from these considerations that $\phi$ can be expanded in the form

$$
\phi=\left(u_{\infty}-1\right) x+\epsilon^{5 / 2} \hat{\phi}_{2}(\hat{x}, y, t)+\ldots \quad \hat{x}=\frac{x-x_{0}}{(K \epsilon)^{1 / 2}}
$$

Retaining the largest terms in Eq. (6) gives Laplace's equation

$$
\hat{\phi}_{2 \hat{x} \dot{x}}+\hat{\phi}_{2 y y}=0
$$

For simplicity the wall shapes are taken to be symmetric near the edges, so that $f_{u}^{\prime \prime}\left(x_{0}\right)=f_{\ell}^{\prime \prime}\left(x_{0}\right)=f^{\prime \prime}\left(x_{0}\right)$. The boundary conditions at the interior walls, for $0<\hat{x}<\infty$, are then

$$
\hat{\phi}_{2 y}(\hat{x}, \pm 1, t)=\mp K^{1 / 2} \hat{x} f^{\prime \prime}\left(x_{0}\right)
$$

and along the exterior surfaces $\hat{\phi}_{2 y}(\hat{x}, \pm 1, t)=0$ for $0<\hat{x}<\infty$. The leading-edge solution [Eq. (26)] must match asymptotically with the channel solution [Eq. (14)]; that is, the expansion of Eq. (26) as $\hat{x} \rightarrow \infty$ is required to agree term by term with the expansion of Eq. (14) as $x \rightarrow x_{0}$. Finally, as $\hat{x}^{2}+y^{2} \rightarrow \infty$ outside the duct, the velocity perturbations must approach zero.

The solution for $\hat{\phi}_{2}$ can be found with the help of a conformal transformation from the complex $\hat{z}$ plane, where $\hat{z}=\hat{x}+i y$, to a complex $\zeta$ plane:

$$
\hat{z}=\frac{1}{\pi}\left(\zeta^{2}-l\right)-\frac{2}{\pi} \ln \zeta+i
$$

The flow is thereby mapped onto the upper half of the $\zeta$ plane. The leading edges $\hat{z}= \pm \mathrm{i}$ are mapped onto the points $\zeta= \pm 1$; near these points $\hat{z} \mp i \sim(2 / \pi)(\zeta \mp 1)^{2}$. As $\hat{z} \rightarrow \infty$ inside the duct, $\zeta \rightarrow 0$ and $\hat{z} \sim-2 \pi^{-1} \ln \xi+i-\pi^{-1}$; as $\hat{z} \rightarrow \infty$ outside the duct, $\zeta \rightarrow \infty$ and $\hat{z} \sim \pi^{-I} \zeta^{2}$. Differentiation with respect to $\hat{x}$ leads to a simpler boundary-value problem, since $\hat{\phi}_{2 \hat{x} y}$ is constant along the walls. Solving this problem for the complex velocity in the $\zeta$ plane and then integrating, using $\mathrm{d} \hat{z} / \mathrm{d} \zeta=2 \pi^{-!}(\zeta$ $-\zeta^{-1}$ ) and adding a source term at the origin $\zeta=0$ to allow matching with the channel solution, one finds the complex velocity

$$
\begin{aligned}
& \hat{\phi}_{2 \bar{x}}-i \hat{\phi}_{2 y}=\frac{1}{\pi^{2}} K^{1 / 2} f^{\prime \prime}\left(x_{0}\right)\left\{1+\left(\zeta^{2}-1\right) \ln \left(1-\frac{1}{\zeta^{2}}\right)\right. \\
& \left.+2 \int_{\zeta}^{\infty} \frac{1}{\zeta} \ln \left(1-\frac{1}{\zeta^{2}}\right) \mathrm{d} \zeta\right\}+\frac{K^{1 / 2}\left\{\pi^{-2} f^{\prime \prime}\left(x_{0}\right)+m_{2}(t)\right\}}{2\left(\zeta^{2}-1\right)}
\end{aligned}
$$

The matching condition as $\hat{x} \rightarrow \infty$, with $-1<y<1$, is found to give

$m_{2}(t)=2 u_{2 \infty}-\left(1-\frac{2}{3} \gamma\right) u_{1 \infty}^{2}-\frac{2}{(\gamma+I) u_{l \infty}}\left\{c_{2 u}(t)+G\left(x_{0}, t\right)\right\}$
Here $u_{l \infty}, u_{2 \infty}$, and $G\left(x_{0}, t\right)$ are considered to be given quantities, and $c_{2 u}(t)$ is obtained from Eq. (21).

At the edges $\hat{z}= \pm i$, the entrance solution for the velocity has the inverse square root singularity associated with flow around a sharp edge. A positive or negative value of $m_{2}(t)+\pi^{-2} f^{\prime \prime}\left(x_{0}\right)$ in Eq. (30) would imply a local outflow or inflow, respectively, around the edges. Since $f^{\prime \prime}\left(x_{0}\right)>0$, an outflow around the edges remains even if the source strength $m_{2}(t)$ is zero. Expansion of the solution [Eq. (30)] as $\zeta \rightarrow \infty$ and $\arg \xi \rightarrow \pi$ shows that the flow ahead of the duct then anticipates the area decrease and accelerates slightly before reaching the duct. Thus a slight contraction of streamtube area occurs ahead of the duct, and the small excess mass flow spills outward around the edges, so that a stagnation point occurs on the inner surface of the wall slightly downstream of the edge.

As $\hat{z} \rightarrow \infty$ outside the duct, the largest term in Eq. (30) is found to be $K^{1 / 2} m_{2}(t) /(2 \pi \hat{z})$, which corresponds to a source of strength $\epsilon^{2} K^{1 / 2} m_{2}(t)$ at $\hat{z}=0$. For the geometry of Fig. 1, an outer solution could be calculated, if desired, in terms of variables $x$ and $(\epsilon K)^{1 / 2} y$, with the largest perturbations described by a velocity potential which satisfies Laplace's equation in terms of these variables. The solution would contain a term which represents a source at $x=0,(\epsilon K)^{1 / 2} y=0$; details are not of special interest here. A local trailing-edge solution also may be necessary. Some types of exit geometry, however, do not require a local flow adjustment at the exit, so that the channel solution remains correct there, as assumed in Eq. (23). For example, the duct might have zero slope and curvature at trailing edges which are joined to fixed parallel walls downstream; or the flow might exhaust into a plenum at $x=x_{1}$, again with $f_{u}^{\prime \prime}\left(\mathrm{x}_{1}\right)=f_{l}^{\prime \prime}\left(x_{l}\right)=0$ and with the flow leaving the edges smoothly, i.e., with the wall streamlines having continuous curvature.

\section{Flow Details near the Throat}

Since the flow accelerates through sonic speed, one might expect that a nonlinear differential equation would be required to describe a mixed flow in the first approximation for some thin region containing the throat. The boundary condition [Eq. (13)] gives $\phi_{y}=\mathcal{O}\left(\epsilon^{2} x\right)$ near the throat if $f^{\prime \prime}(0) \neq 0$. If the terms $\phi_{y y}$ and $(\gamma+1) \phi_{x} \phi_{x x}$ in the differential equation (6) are both of the same order of magnitude, it follows for $y=\mathcal{O}(1)$ that $x=\mathcal{O}(\epsilon), \phi_{x}=\mathcal{O}\left(\epsilon^{2}\right)$, and $\phi_{y}=\mathcal{O}\left(\epsilon^{3}\right)$. The potential then can be expanded in the form

$$
\begin{gathered}
\phi=(\gamma+1)^{1 / 2}\left[-f^{\prime \prime}(0)\right]^{3 / 2} \epsilon^{3} \phi_{2}^{*}\left(x^{*}, y, t\right)+\ldots \\
x^{*}=(\gamma+l)^{-1 / 2}\left[-f^{\prime \prime}(0)\right]^{-1 / 2}(x / \epsilon)
\end{gathered}
$$

The largest terms in Eqs. (6) and (13) give

$$
\begin{gathered}
\phi_{2 y y}^{*}=\phi_{2 x^{*}}^{*} \phi_{2 x^{*} x^{*}}^{*} \\
\phi_{2 y}^{*}\left(x^{*}, \pm 1, t\right)=\left\{ \pm 1-\frac{f_{l}^{\prime \prime}(0)-f_{u}^{\prime \prime}(0)}{2 f^{\prime \prime}(0)}\right\} x^{*} \\
+\left\{\mp I+\frac{G_{\ell x}(0, t)-G_{u x}(0, t)}{2 G_{x}(0, t)}\right\} x_{l}^{*}
\end{gathered}
$$

where

$$
x_{i}^{*}=-(\gamma+1)^{-1 / 2}\left[-f^{\prime \prime}(0)\right]^{-3 / 2} G_{x}(0, t)
$$

If the channel-flow solutions for $\phi_{I x}$ and $\phi_{2 x}$ are evaluated as $x \rightarrow 0$, it can be seen that matching with a solution $\phi_{x}=\Theta\left(\epsilon^{2}\right)$ is possible only if $c_{1}$ and $c_{2 u}$ are given by Eqs. (18) and (21). That is, if these conditions were not met, the velocity would not become so close to the sound speed that a nonlinear flow description is required. In the case considered here, the flow 
accelerates from subsonic to supersonic speed, and a rather simple generalization of the steady-flow polynomial solution (e.g., Ref. 8) gives

$$
\begin{aligned}
\phi_{2}^{*} & =\frac{1}{2}\left(x^{*}-x_{I}^{*}\right)^{2}+\frac{1}{2}\left(x^{*}-x_{l}^{*}\right)\left(\left(y^{2}-\frac{1}{3}\right)+\frac{1}{12}\left(\frac{1}{2} y^{4}-y^{2}\right)\right. \\
& -\frac{1}{2}\left\{f_{\ell}^{\prime \prime}(0)-f_{u}^{\prime \prime}(0)\right\}\left\{x^{*} y+\frac{1}{2}\left(\frac{1}{3} y^{3}-y\right)\right\} / f^{\prime \prime}(0) \\
& +\frac{1}{2}\left\{G_{b x}(0, t)-G_{u x}(0, t)\right\} x_{l}^{*} y / G_{x}(0, t)
\end{aligned}
$$

The velocity components can easily be written in the original variables. The sonic line is given by

$$
\begin{aligned}
x= & \epsilon \frac{G_{x}(0, t)}{f^{\prime \prime}(0)}+\epsilon \frac{I}{2}(\gamma+i)^{1 / 2}\left[-f^{\prime \prime}(0)\right]^{1 / 2} \\
& \times\left\{\frac{1}{3}-y^{2}+\frac{f_{\rho}^{\prime \prime}(0)-f_{u}^{\prime \prime}(0)}{f^{\prime \prime}(0)} y\right\}+\ldots
\end{aligned}
$$

The first term is the value of $x$ at which the instantaneous channel width is a minimum and the remaining terms represent the steady-state sonic-line shape. That is, for the particular limiting case corresponding to $\alpha=\mathcal{O}\left(\epsilon^{3}\right)$ and $\tau^{-1}=\mathcal{O}\left(\epsilon^{2}\right)$, the sonic line is found to have the appropriate quasisteady location at each instant.

A composite solution for $\phi$ can be formed which reduces to Eq. (14) for $x=\mathcal{O}(1)$ and to Eq. (32) for $x=\mathcal{O}(\epsilon)$. It is convenient first to note that, as for steady flow, ${ }^{8}$ an equivalent form of the channel solution is obtained if $f$ and $c_{l}$ are replaced by $f-\epsilon G$ and $c_{1}+\epsilon c_{2}$ in Eq. (18), with the term $(\gamma+1)^{-1}\left(c_{2}+G\right) / \phi_{1 x}$ omitted in Eq. (20). Then,

$$
u=I \pm \epsilon[2 /(\gamma+I)]^{1 / 2}\left\{c_{1}-f(x)+\epsilon c_{2}(t)+\epsilon G(x, t)\right\}^{1 / 2}+\ldots
$$

The equivalence, up to $\mathcal{O}\left(\epsilon^{2}\right)$, is shown by expansion of the resulting square root in Eq. (37). This solution is, in fact, a composite solution which contains a simple inner solution with $\phi_{x}=\mathcal{O}\left(\epsilon^{3 / 2}\right)$ when $x=\mathcal{O}\left(\epsilon^{1 / 2}\right)$, and thus can include a flow with velocity at the throat which differs from the sound speed by $\mathcal{O}\left(\epsilon^{3 / 2}\right)$. One more modification is required to obtain a solution which can describe the choked condition as well. If the solution is required to be correct only through terms of order $\epsilon^{2}$, it is uniquely determined only up to that order, and higher order details will depend on the method used to construct the solution. Perhaps the simplest choice gives

$$
\begin{aligned}
u= & I \pm \epsilon\left(\frac{2}{\gamma+1}\right)^{1 / 2}\left\{c_{l}-f(x)+\epsilon c_{2}(t)+\epsilon G(x, t)\right. \\
& \left.+\frac{\epsilon^{2} G_{x}^{2}(0, t)}{2\left[-f^{\prime \prime}(0)\right]}\right\}^{1 / 2}+\epsilon^{2}\left\{\frac{1}{2} f^{\prime \prime}(x)\left(\frac{1}{3}-y^{2}\right)\right. \\
& \left.+\frac{1}{2}\left[f_{l}^{\prime \prime}(x)-f_{u}^{\prime \prime}(x)\right] y+\left(\frac{1}{2}-\frac{\gamma}{3}\right) \frac{2}{\gamma+1}\left[c_{l}-f(x)\right]\right\}+\ldots
\end{aligned}
$$

where, again, the upper and lower signs refer to supersonic and subsonic flow, respectively. Equation (38) is correct to order $\epsilon^{2}$ both near and away from the throat, since it may be expanded for $x=\mathcal{\Theta}(\epsilon)$ to give the throat solution and for $x=\mathcal{O}(1)$ to give the channel solution. Moreover, the case $c_{2}(t)+G(0, t)>0$ is also included, since then Eq. (37) is recovered by expansion of Eq. (38) for $x=\mathcal{O}\left(\epsilon^{1 / 2}\right)$.
For flows accelerating through sonic speed at the throat, a comparison of the channel and throat solutions shows that the velocity perturbations $\epsilon \mathcal{u}_{1}+\epsilon^{2} u_{2}$ and $\epsilon^{2} v_{2}$ found from Eqs. (14), (18), and (19) do in fact remain correct up to $\mathcal{O}\left(\epsilon^{2}\right)$ as $x \rightarrow 0$, and so the nonlinear equation is not really needed. The composite solution [Eq. (38)] perhaps offers a better form for $u$ since the indeterminate form $\left(c_{2}+G\right) / \phi_{I x}$ is avoided. However, if the velocity approaches very close to the sound speed but is never exactly sonic, the outer solution does not give the correct flow details near the throat, whereas Eq. (38) does remain correct.

\section{Velocity and Position of the Shock Wave}

The jump in the velocity across the shock wave at $x=x_{s}(y, t)$ is in a direction normal to the shock, and so $\partial x_{s} / \partial y$ $=-\left(v_{d}-v_{u}\right) /\left(u_{d}-u_{u}\right)$. If it is assumed tentatively that $v_{d}-v_{u}=o\left(\epsilon^{2}\right)$, then the first two terms in the expansion of $x_{s}$ are independent of $y$ :

$$
x_{s}(t, y)=x_{s 0}(t)+\epsilon x_{s l}(t)+\ldots
$$

Since $u_{s}=\tau^{-1} \partial x_{s} / \partial t$, substitution of Eq. (39) into Eq. (12) gives

$$
\begin{aligned}
& u_{I d}=-u_{l u} \\
& u_{2 d}=-u_{2 u}-u_{I d} u_{l u}+\frac{4}{\gamma+1} k \frac{\mathrm{d} x_{s 0}}{\mathrm{~d} t}
\end{aligned}
$$

Equation (40a) is satisfied by Eq. (18), but the solution [Eq. (19)] for $\phi_{2}$ shows a dependence on $y$ and so Eq. (40b) cannot be satisfied by $u_{2}=\phi_{2 x}$. As in earlier work, ${ }^{1,3,4,8}$ an adjustment of the flow takes place within a short distance downstream of the shock wave. A correction term $\epsilon^{5 / 2}(\gamma+1)^{1 / 2} u_{l u}^{1 / 2} \tilde{\phi}_{2}(\tilde{x}, y, t)$, where $\tilde{x}=\left(x-x_{s}\right) /\left[\epsilon(\gamma+1) u_{l u}\right]^{1 / 2}$, is added to the expansion [Eq. (14)]; $\tilde{\phi}_{2}$ satisfies Laplace's equation in the semi-infinite strip $0<\tilde{x}<\infty,-1<y<1$, subject to the boundary conditions $\tilde{\phi}_{2 \bar{x}}(0, y, t)=\left(y^{2}-\right.$ $1 / 3) f^{\prime \prime}\left(x_{s 0}\right)-2 y g^{\prime}\left(x_{s 0}\right), \tilde{\phi}_{2 y}(\tilde{x}, \pm 1, t)=0$, and $\widetilde{\phi}_{2 \tilde{x}} \rightarrow 0$ as $\tilde{x} \rightarrow \infty$. The result is

$$
\begin{aligned}
\tilde{\phi}_{2} & =-\frac{4}{\pi^{3}}\left\{f^{\prime \prime}\left(x_{s 0}\right) \sum_{n=1}^{\infty} \frac{(-1)^{n}}{n^{3}} \exp (-n \pi \tilde{x}) \cos n \pi y\right. \\
& \left.\left.+8 g^{\prime}\left(x_{s 0}\right) \sum_{n=1}^{\infty} \frac{(-1)^{n}}{(2 n-1)^{3}} \exp \left(-\frac{2 n-1}{2} \pi \tilde{x}\right)\right) \sin \frac{2 n-1}{2} \pi y\right\}
\end{aligned}
$$

for $x>x_{s}$; no correction is needed for $x<x_{s}$. The addition of this correction to Eq. (14) provides a composite solution which is valid everywhere except near the duct entrance and perhaps near the exit, where a solution of the type given in Eq. (26) is needed. As already noted, the solution for the velocity also can be modified in the manner of Eq. (38). It follows, moreover that $\partial x_{s} / \partial y=\mathcal{O}\left(\epsilon^{3 / 2}\right)$, consistent with the assumption of a nearly normal shock wave in Eqs. (11), (12), and (39).

It can be seen from the results derived thus far that the velocity and pressure are everywhere described by quasisteady solutions since $t$ enters these solutions only as a parameter. The shock-wave motion, however, is not quasisteady since $u_{s}$ rather than $x_{s}$ depends on instantaneous conditions. Equations (40b), (21), and (24) now give, with the contribution of $\widetilde{\phi}_{2}$ included in $u_{2 d}$,

$$
\begin{gathered}
\frac{\mathrm{d} x_{s 0}}{\mathrm{~d} t}=-\frac{1}{4 k u_{l u}}\left[\frac{2}{3} \gamma(\gamma+l) u_{l u}^{3}+N(t)\right] \\
N(t)=c_{2 d}-c_{2 u}=G(0, t)-G\left(x_{l}, t\right)+c_{2 d}^{(s)}+G_{d}(t)
\end{gathered}
$$


Since $u_{1 u}=\left\{2\left[f(0)-f\left(x_{s 0}\right)\right] /(\gamma+1)\right\}^{1 / 2}$, the right-hand side of Eq. (42a) is a known function of $x_{s 0}$ and $t$. Equation (42a) is a nonlinear differential equation for the shock-wave position $x_{s 0}(t)$, similar to that studied in Ref. 3 . Here, however, $x_{s 0}(t)$ depends not only on the downstream pressure characterized by $c_{2 d}^{(s)}+G_{d}(t)$, but also on the difference $G(0, t)-G\left(x_{1}, t\right)$ in the wall displacements at the throat and exit. Clearly there is an equivalence between these two functions, since the shockwave motion depends only on their sum. A given shock-wave motion might be caused by wall oscillations, by oscillations in downstream pressure, or by some combination of the two. However, for a given shock-wave motion the velocity and pressure distribution, of course, will depend on the source of the disturbances. These quantities depend on the instantaneous values of downstream pressure and wall location; a dependence on $x$ appears for wall oscillations but not for oscillations in downstream pressure.

At values of $t$ when $G_{d}(t)>0$, the exit pressure is greater than its steady-state value, and the equilibrium position of the shock wave is shifted upstream. The actual shock-wave location tends to follow the equilibrium position, but lags somewhat behind. In Eq. (42a) for the shock-wave velocity, the term proportional to $G_{d}(t)$ is negative when $G_{d}(t)>0$, giving a negative contribution to $\mathrm{d} x_{s 0} / \mathrm{d} t$ as expected. An equivalent effect is found at values of $t$ when $G\left(x_{l}, t\right)<G(0, t)$, i.e., when a slight convergence of the walls is added to the steady-state wall shapes. If the exit pressure is unchanged, the pressure at points between the shock wave and the exit is increased, because the ratio of the local cross-sectional area to the exit area is increased. Changes in the location of the center of pressure on the wall are primarily the result of the changes in size of the regions of subsonic and supersonic flow which occur because of the shock-wave motion. If, for example, the walls undergo symmetric pitching oscillations about $x=0$, for constant exit pressure, because of the phase lag the maximum upstream displacement of the shock wave and the maximum size of the downstream subsonic region occur at times when the walls are moving outward for $x>0$, and the minima occur when the walls are moving inward. Thus the time-dependent pressure force on the walls has a component in phase with the velocity of the walls, which would tend to amplify the motion. These relationships would be simplified further if one were to assume an intermediate case such that $\epsilon^{2} \ll \tau^{-1} \ll \epsilon$, again with $\alpha=\epsilon^{3}$, corresponding to slightly faster oscillations so that the oscillating shock-wave displacement has smaller amplitude $\mathcal{O}\left(\tau \epsilon^{2}\right)$. For simple harmonic wall oscillations with $G=\beta_{0} e^{i t} x$, the nonlinear differential equation (42a) for the shock-wave position would be replaced by the simple linear equation $\mathrm{d} x_{s} / \mathrm{d} t=1 / 4 \tau \epsilon^{2} \beta_{0} e^{i t} x_{1} / u_{I u}+\ldots$, where $u_{l}$ is evaluated at the steady-state shock-wave location $x=x_{s}^{(s)}$. It would follow that $x_{s}(t)=x_{s}^{(s)}-1 / 4 i \tau \epsilon^{2} \beta_{0} e^{i t} x_{I} / u_{l u}+\ldots$, and the first term in the oscillating pressure force would be exactly in phase with the velocity of the walls.

\section{Motion of the Shock Wave Upstream of the Throat}

If the shock wave approaches the throat as the time $t$ approaches a value $t_{*}$, clearly $\mathrm{d} x_{s 0} / \mathrm{d} t \rightarrow \infty$ in Eq. (42a), since $u_{I u} \rightarrow 0$, where $u_{I u} \sim(\gamma+1)^{-1 / 2}\left[-f^{\prime \prime}(0)\right]^{1 / 2} x_{s 0}$. Comparison of the expressions for $\mathrm{d} x_{s 0} / \mathrm{d} t$ and $u_{l u}$ suggests, as in Ref. 3 , that the shock-wave speed $u_{s}$ and the perturbation $u-1$ in the fluid velocity are both $\mathcal{O}\left(\epsilon^{3 / 2}\right)$ when $x_{s}=\mathcal{O}\left(\epsilon^{1 / 2}\right)$ and $t-t_{*}=\mathcal{O}(\epsilon)$. That is, the shock wave passes through a thin region $x=\mathcal{O}\left(\epsilon^{1 / 2}\right)$ at a nearly constant value of $t$, namely at $t \approx t_{*}$, where $t_{*}$ is considered to be known from the behavior of the solution for $x_{s 0}(t)$ as $x_{s 0} \rightarrow 0$. Equation (12) becomes

$$
u_{s}=[(\gamma+1) / 4]\left\{\left(u_{u}-1\right)+\left(u_{d}-l\right)\right\}+\ldots
$$

where $u_{u}-1=\epsilon(\gamma+1)^{-1 / 2}\left[-f^{\prime \prime}(0)\right]^{1 / 2} x_{s}+\ldots$. Downstream of the shock wave $u-1$ can be found from the composite solution [Eq. (37)] with $c_{2}=c_{2 d}$. Setting $x=x_{s}$ and expanding as $x_{s} \rightarrow 0$, one finds

$u_{d}-l=-\epsilon^{3 / 2}\left(\frac{2}{\gamma+1}\right)^{1 / 2}\left\{-\frac{x_{s}^{2}}{2 \epsilon} f^{\prime \prime}(0)+N\left(t_{*}\right)\right\}^{1 / 2}+\mathcal{O}\left(\epsilon^{2}\right)$

where $N\left(t_{*}\right)>0$. The shock-wave position can be expressed in the form

$$
\begin{aligned}
& x_{s}=\epsilon^{1 / 2}\left[-2 N\left(t_{*}\right) / f^{\prime \prime}(0)\right]^{1 / 2} \xi_{s l}\left(t^{*}\right)+\ldots \\
& t^{*}=(1 / 4 k)(\gamma+1)^{1 / 2}\left[-f^{\prime \prime}(0)\right]^{1 / 2}\left(t-t_{*}\right) / \epsilon
\end{aligned}
$$

Substitution in Eq. (43) leads to a differential equation for $\xi_{s I}$ which can be solved implicitly:

$$
\begin{gathered}
\frac{\mathrm{d} \xi_{s l}}{\mathrm{~d} t^{*}}=\xi_{s I}-\left(1+\xi_{s l}^{2}\right)^{1 / 2} \\
2 t^{*}=1-\left(1+\xi_{s l}^{2}\right)^{1 / 2}\left\{\xi_{s l}+\left(1+\xi_{s l}^{2}\right)^{1 / 2}\right\}-\ln \left\{\xi_{s l}+\left(1+\xi_{s l}^{2}\right)^{1 / 2}\right\}
\end{gathered}
$$

The integration constant has been chosen so that $\xi_{s l}=0$ when $t^{*}=0$. The matching condition as $x_{s 0} \rightarrow 0$ and $\xi_{s l} \rightarrow \infty$ requires that the largest terms in Eqs. (39) and (45a) for $x_{s}$ be identical when written in common notation. As $\xi_{s l} \rightarrow \infty$ and $t^{*} \rightarrow-\infty$, the solution [Eq. (46b)] gives $\xi_{s I} \sim\left(-t^{*}\right)^{1 / 2}$, in agreement with the largest term in $x_{s 0}$ found by integration of Eq. (42a) as $u_{1 u} \rightarrow 0$.

As the shock wave moves upstream from the throat, again $x_{s}=\mathcal{O}(1)$, and now the shock-wave speed $u_{s}=\mathcal{O}(\epsilon)$, so that the relative velocity $u_{u}-u_{s}$ can be supersonic. Still another representation of $x_{s}(t)$ is needed, which must match with the expansion of Eq. (46b) as $\xi_{s t} \rightarrow-\infty$ and $t^{*} \rightarrow \infty$. In this limit it is found that $\ln \left(-2 \xi_{s l}\right) \sim 2\left[t^{*}-(1 / 4)\right]$. Rewriting this result in the original variables suggests that $t^{*}=0[\ln (1 / \epsilon)]$ when $x_{s}=\mathcal{O}(1)$. For proper matching the new representation must have the form

$$
\begin{aligned}
& x_{s}=x_{s 0}^{+}\left(t^{+}\right)+\ldots \\
& t^{+}=\frac{1}{k \epsilon}\left(t-t_{*}\right)-\frac{\ln (1 / \epsilon)}{(\gamma+1)^{1 / 2}\left[-f^{\prime \prime}(0)\right]^{1 / 2}}
\end{aligned}
$$

The flow is subsonic both ahead of and behind the shock wave, with $u_{l d}=u_{l u}<0$ found from Eq. (18). Substitution in Eq. (43) gives a differential equation for $x_{s 0}^{+}$:

$$
\frac{\mathrm{d} x_{s 0}^{+}}{\mathrm{d} t^{+}}=-\left(\frac{\gamma+I}{2}\right)^{1 / 2}\left\{f(0)-f\left(x_{s 0}^{+}\right)\right\}^{1 / 2}
$$

Since $f(0)-f\left(x_{s 0}^{+}\right) \sim-1 / 2 x_{s 0}^{+2} f^{\prime \prime}(0)$ as $x_{s 0}^{+} \rightarrow 0$, the solution as $x_{s 0}^{+} \rightarrow 0$ is

$x_{s 0}^{+}=-\left[\frac{N\left(t_{*}\right)}{-2 e f^{\prime \prime}(0)}\right]^{1 / 2} \exp \left\{\frac{1}{2}(\gamma+I)^{1 / 2}\left[-f^{\prime \prime}(0)\right]^{1 / 2} t^{+}\right\}+\ldots$

where the multiplicative factor is an integration constant chosen for matching with the expansion of Eq. (46b) as $\xi_{s t} \rightarrow-\infty$. Equation (49) provides an initial condition for the differential equation (48).

The simplest special case arises for a duct of length $2 x_{I}$ with $x_{0}=-x_{I}$ having symmetric parabolic walls described by $f(0)-f(x)=\left(x / x_{l}\right)^{2} f(0)$, where $f\left( \pm x_{I}\right)=0$ and the first-order choking condition [Eq. (25)] gives $f(0)=1 / 2(\gamma+1) u_{1 \infty}^{2}$. Integration of the shock-wave velocity [Eq. (48)] shows that the 
solution [Eq. (49)], with $f^{\prime \prime}(0)=-(\gamma+1) u_{l \infty}^{2} / x_{l}^{2}$, remains correct for the entire shock-wave motion from the throat $x=0$ up to the entrance $x=-x_{I}$. The time required for passage of the shock wave through this region is found to be

$$
t-t_{*}=\frac{k x_{I} \epsilon}{(\gamma+1)\left(-u_{l \infty}\right)} \ln \frac{2(\gamma+1) u_{l \infty}^{2} e}{\epsilon N\left(t_{*}\right)}
$$

The parabolic wall shape, however, implies wedge-shaped leading and trailing edges, since $f^{\prime}\left( \pm x_{l}\right) \neq 0$. A simple shape having cusped edges is given by $f(0)-f(x)=f(0) \sin ^{2}$ $\times\left(1 / 2 \pi x / x_{l}\right)$, where again $f\left( \pm x_{l}\right)=0$ and $f(0)=1 / 2(\gamma+1) u_{l \infty}^{2}$. Equation (48) can be integrated, and the integration constant is found from Eq. (49) as $x_{s 0}^{+} \rightarrow 0$, now with $f^{\prime \prime}(0)=$ $-1 / 4 \pi^{2}(\gamma+1) u_{l \infty}^{2} / x_{1}^{2}$. The shock-wave position and the time required for the shock wave to move upstream through the front half of the duct are found as

$$
\begin{gathered}
\tan \frac{\pi x_{s 0}^{+}}{4 x_{I}}=-\left[\frac{N\left(t_{*}\right)}{8(\gamma+1) e u_{l \infty}^{2}}\right]^{1 / 2} \exp \left\{\frac{(\gamma+I) \pi\left(-u_{I \infty}\right) t^{+}}{4 x_{I}}\right\} \\
t-t_{*}=\frac{2 x_{1} k \epsilon}{(\gamma+I) \pi\left(-u_{1 \infty}\right)} \ln \frac{8(\gamma+I) e u_{l \infty}^{2}}{\epsilon N\left(t_{*}\right)}
\end{gathered}
$$

The results, Eqs. (50) and (51b), for different shapes show identical dependence on the parameters; only the numerical factors are different.

In Refs. 3 and 4, numerical calculations of the shock-wave position had to be terminated when the shock wave reached the throat. These calculations now can be continued to include the motion upstream from the throat. For an arbitrary wall shape, the shock-wave velocity $u_{s}=\tau^{-1} \partial x_{s} / \partial t$ is found from Eqs. (39) and (42) for $x_{s}=\mathcal{O}(1)$ downstream of the throat, from Eqs. (45) and (46) for $x_{s}=\mathcal{O}\left(\epsilon^{1 / 2}\right)$, and from Eqs. (47) and (48) for $x_{s}=\mathcal{O}(1)$ upstream of the throat. It is probably most convenient to replace these three representations by two composite expressions, one for $x_{s} \geq 0$ and one for $x_{s} \leq 0$, each uniformly valid to order $\epsilon^{2}$. This formulation can be accomplished for $x_{s} \geq 0$ by adding the downstream and throat representations, and then subtracting the common part found for $x_{s} \ll 1$ and $x_{s} \gg \epsilon^{1 / 2}$; for $x_{s}<0$ the upstream and throat solutions are added, and the common part for $\left|x_{s}\right| \ll 1$ and $\left|x_{s}\right|>\epsilon^{1 / 2}$ is subtracted. The result for $x_{s}>0$, a generalization of that given in Ref. 3 , is

$$
\begin{aligned}
u_{s} & =-\epsilon^{2} \frac{\gamma}{3}\left[f(0)-f\left(x_{s}\right)\right]+\epsilon^{3 / 2} \frac{(\gamma+I)^{1 / 2}}{4}\left[-f^{\prime \prime}(0)\right]^{1 / 2} \\
& \times\left\{\frac{x_{s}}{\epsilon^{1 / 2}}-\left[\frac{x_{s}^{2}}{\epsilon}-\frac{2 N(t)}{f^{\prime \prime}(0)}\right]^{1 / 2}\right\}+\frac{\epsilon^{2}}{4}\left(\frac{\gamma+1}{2}\right)^{1 / 2} \\
& \times N(t)\left\{\frac{2^{1 / 2}}{x_{s}\left[-f^{\prime \prime}(0)\right]^{1 / 2}}-\frac{1}{\left[f(0)-f\left(x_{s}\right)\right]^{1 / 2}}\right\}+\ldots
\end{aligned}
$$

and for $x_{s}<0$ one finds

$$
\begin{aligned}
u_{s} & =-\epsilon\left(\frac{\gamma+1}{2}\right)^{1 / 2}\left[f(0)-f\left(x_{s}\right)\right]^{1 / 2}-\epsilon^{3 / 2} \frac{(\gamma+1)^{1 / 2}}{4} \\
& \times\left[-\frac{x_{s}^{2}}{\epsilon} f^{\prime \prime}(0)+2 N(t)\right]^{1 / 2}-\epsilon \frac{(\gamma+1)^{1 / 2}}{4}\left[-f^{\prime \prime}(0)\right]^{1 / 2} x_{s}+\ldots
\end{aligned}
$$

These results describe the effects of oscillations in downstream pressure as characterized by $G_{d}(t)$, or in wall shape for an equivalent $G(x, t)$. By numerical integration of Eq. (53), the calculations for cases a and $c$ in Ref. 4, Fig. 15, have been extended in Ref. 7 to include the entire motion of the shock wave.

\section{Numerical Examples}

Numerical solutions have been carried out for a symmetric channel with shape defined by

$$
\begin{aligned}
f(x) & =3-\frac{18}{13} x^{2} & & 0 \leq x \leq 1 \\
& =-\frac{27}{13}(x-2)^{4}-\frac{48}{13}(x-2)^{3} & & 1 \leq x \leq 2 \\
& =0 & & x \geq 2
\end{aligned}
$$

It can be seen that $f^{\prime \prime}(x)<0$ for $0<x<10 / 9, f^{\prime \prime}(x)>0$ for $10 / 9<x<2$, and $f^{\prime \prime}(x)$ is continuous everywhere. Two cases were considered in Ref. 7 , both with the same shock-wave motion. In one case the walls were stationary and the downstream pressure was specified by setting

$$
G_{d}(t)=3(\gamma+1) \sin 2 t
$$

for $t>0$ and $G_{d}(t)=0$ for $t<0$. In the other case the downstream pressure was constant and a wall oscillation was prescribed by setting

$$
G(x, t)=-\left[\left(x-x_{c}\right) / x_{l}\right] 3(\gamma+1) \sin 2 t
$$

for $t>0$ and $G(x, t)=0$ for $t<0$. The parameters were taken as $\epsilon=0.1, \tau^{-1}=0.01, \gamma=1.4$, and $x_{1}=3$. The value of $x_{c}$ does not enter the solution; one could, e.g., consider $x_{c}=3$, with $G(x, t)=0$ for $x>3$, and local corrections associated with the jump in $G_{x}$ at $x=3$ would be of higher order. The steady-state shock-wave position is $x_{s 0}^{(s)}=1.5$ in both cases, and $c_{c d}^{(s)}$ is then given $^{8}$ by $c_{2 d}^{(s)}=-2 / 3 \gamma(\gamma+1)\left(u_{u}^{(s)}\right)^{3}$, where $u_{u}^{(s)}$ is found from Eq. (18) with $x=x_{s 0}^{(s)}$.

The shock-wave position $x_{s 0}(t)$ is calculated by numerical integration of Eq. (42), and the velocities are found from the expansion [Eq. (14)] with the correction $\epsilon^{5 / 2} \tilde{\phi}_{2}$ from Eq. (41) added. The parameters are such that the shock wave always remains downstream of the throat. For the case of oscillating walls with constant downstream pressure, lines of constant velocity are shown in Fig. 2 at $t=\pi / 2$ and in Fig. 3 at $t=3 \pi / 4$. Pressures at the wall and at the centerline are also shown. The same quantities are plotted in Ref. 7, Fig. 6, for the case of stationary walls and oscillating back pressure; the differences from the present Figs. 2 and 3 occur only in the second approximation and, therefore, are small.
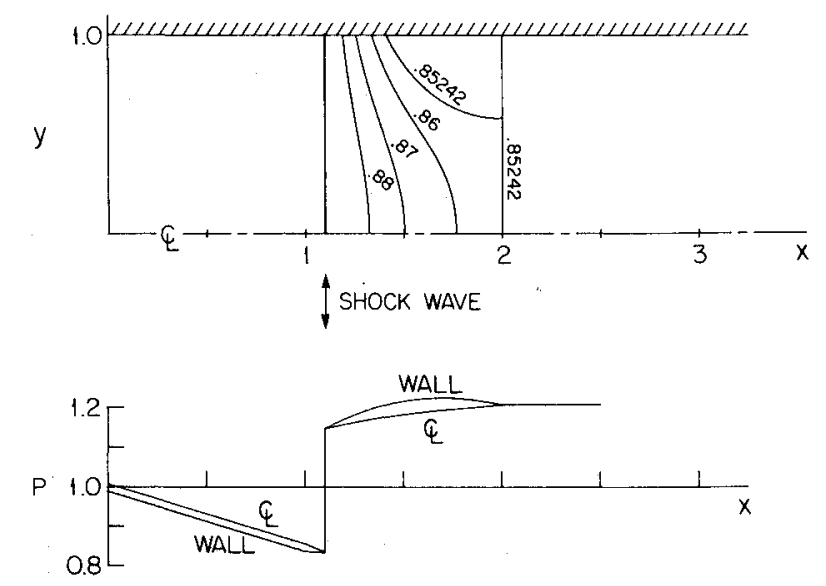

Fig. 2 Constant-velocity contours and pressure distributions at wall and centerline for conditions of Eqs. (54) and (56) at $t=\pi / 2$. 
In Fig. 2, for $t=\pi / 2$, the shock wave lies very close to the location $x=10 / 9$ where the wall curvature is zero, and the inner solution [Eq. (41)] is negligible. The wall and centerline pressures are equal at the shock wave, and the streamline curvature at any other value of $x$ has the same sign as the local wall curvature. In Fig. 3 , at $t=3 \pi / 4$, this is no longer true. Behind the shock wave the reversal of the transverse pressure gradient, associated with a normal shock wave at a curved surface, is evident since the wall pressure remains lower than the centerline pressure in a region where $f^{\prime \prime}(x) \geq 0$. The influence of the inner solution [Eq. (41)] extends over a considerable distance, as would be expected since the length scale is $\mathcal{O}\left(\epsilon^{1 / 2}\right)$ and here $\epsilon^{1 / 2} \approx 0.3$. The logarithmic singularity in $\partial p / \partial x$, which is contained in Eq. (41), is not evident because calculations were not carried out at points sufficiently close to the foot of the shock wave. In each figure the small velocity changes, of course, would be increased if the area change were larger. The irregularities at $x=2$ arise because of the discontinuity in wall curvature at $x=2$, and would be absent if the curvature were continuous.

\section{Solutions for More Rapid Oscillations}

If the ratio $\tau=\omega^{-1} a^{*} / L=\mathcal{O}\left(\epsilon^{-1}\right)$, downstream disturbances no longer reach the shock wave in a time which is short in comparison with the characteristic time of the oscillation. These disturbances travel upstream at a speed $|u-a|=\mathcal{O}(\epsilon)$, with varying amplitude which at each point depends on the local cross-sectional area. For the case of oscillating walls, the sonic line near the throat no longer has a quasisteady position but instead lags behind the wall motion. Since the flow is onedimensional in a first approximation for wall shapes given by Eq. (1), the steady-state gas velocity is $1+\vartheta(\epsilon)$, and the largest time-dependent term in $u$ is $\mathcal{O}(\alpha / \epsilon)$. The equivalent downstream pressure fluctuation is $\mathcal{O}(\alpha / \epsilon)$, the shock-wave velocity is $\mathcal{O}(\alpha / \epsilon)$, and the shock-wave displacement is $\mathcal{O}(\tau \alpha / \epsilon)$. In this section two cases are considered briefly: $\alpha=\mathcal{O}\left(\epsilon^{2}\right)$ and $\alpha=\mathcal{O}\left(\epsilon^{3}\right)$.

\section{Small-Amplitude Oscillations}

For $\tau^{-1}=k \epsilon$ and $\alpha=\epsilon^{3}$, an expansion of the form of Eq. (14) for the channel flow again leads to Eqs. (18) and (19), where $h_{2 x}$ now satisfies

$$
\begin{aligned}
& 2 k h_{2 x l}+(\gamma+l)\left(\phi_{I x} h_{2 x}\right)_{x} \\
& \quad=(\gamma+I)\left[\phi_{I x} \frac{f^{\prime \prime}}{6}-\frac{2 \gamma-3}{6} \phi_{I X}^{3}+\frac{G}{(\gamma+1)}\right]_{x}
\end{aligned}
$$
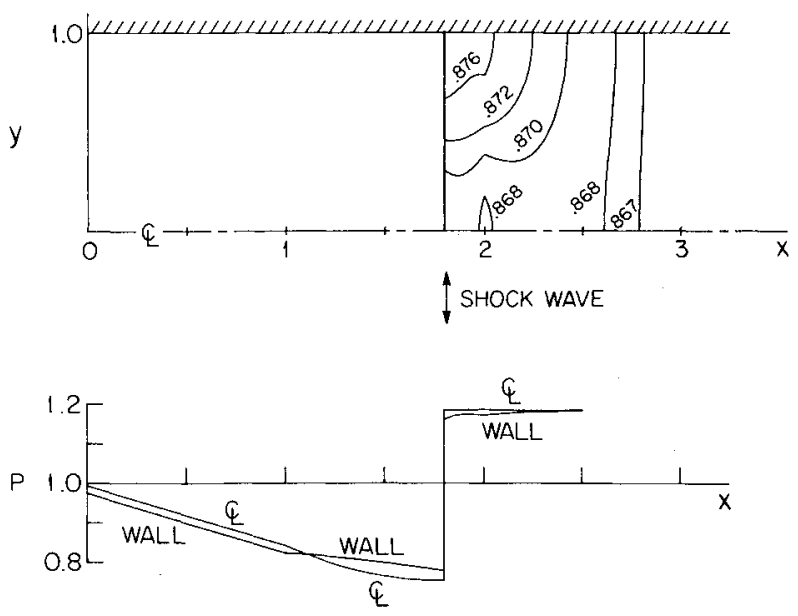

Fig. 3 Constant-velocity contours and pressure distributions at wall and centerline for conditions of Eqs. (54) and (56) at $t=3 \pi / 4$.
For the flow downstream of the shock wave, effects of oscillations in back pressure and in wall shape can be separated if the solution for $h_{2 x}$ is written in the form

$$
\begin{gathered}
\phi_{l x} h_{2 x}=\phi_{l x} \frac{f^{\prime \prime}}{6}-\frac{2 \gamma-3}{6} \phi_{l x}^{3}+R(x, t) \\
+\frac{1}{\gamma+1} G_{d}\left(t-\frac{2 k}{\gamma+1} \int_{x_{l}}^{x} \frac{\mathrm{d} x}{\phi_{l x}}\right)
\end{gathered}
$$

Here $G_{d}(t)$ is again defined by Eqs. (23) and (24); $R(x, t)$ contains the effects of the wall oscillations and satisfies

$$
2 k R_{t}+(\gamma+1) \phi_{I x} R_{x}=\phi_{I x} G_{x}
$$

In general Eq. (59) would be solved by numerical integration along characteristics $\mathrm{d} x / \mathrm{d} t=(\gamma+1) \phi_{I X} /(2 k)$, with $R\left(x_{1}, t\right)=(\gamma+1)^{-1} c_{2 d}^{(s)}$. For the flow upstream of the shock wave, the last term in Eq. (58) is absent, and Eq. (59) is integrated with the requirement that $R(x, t) \rightarrow 0$ as $x \rightarrow 0$ in such a way that the solution matches correctly with the local solution near the throat.

For $x=\mathcal{O}(\epsilon)$, the potential again has the form of Eq. (32), with the exception that the differential equation (33a) has an added term $2 k(\gamma+1)^{-1 / 2}\left\{-f^{\prime \prime}(0)\right\}^{-1 / 2} \phi_{2 x^{*} t}^{*}$ on the right-hand side. A particular form of solution to this equation was considered in Ref. 16. In the present case, the solution for $\phi_{2}^{*}$ is again a polynomial in $x^{*}$ and $y$, but the term $-x_{1}^{*}$ in $\phi_{2 x^{*}}^{*}$ now is replaced by an arbitrary function of time, say, $-\lambda(t) / f^{\prime \prime}(0)$, which satisfies the simple differential equation

$$
2 k \frac{\mathrm{d} \lambda}{\mathrm{d} t}+(\gamma+1)^{1 / 2}\left\{-f^{\prime \prime}(0)\right\}^{1 / 2} \lambda=G_{x}(0, t)
$$

In the expansion equation (39) for the shock-wave location, the term $x_{50}$ is now constant, and the amplitude of the shockwave displacement is $\mathcal{O}(\epsilon)$. The correction term $\tilde{\phi}_{2}$ for the flow immediately downstream of the shock wave is again given by Eq. (41). Substitution of the solutions for $u_{u}$ and $u_{d}$ into Eq. (12) gives finally

$$
\frac{4 k}{\gamma+l} \frac{\mathrm{d} x_{s I}}{\mathrm{~d} t}=\left(h_{2 x}\right)_{d}+\left(h_{2 x}\right)_{u}-\frac{1}{3} f^{\prime \prime}(0)-u_{1 u}^{2}
$$

where the subscripts $u$ and $d$ indicate values immediately upstream and downstream of the steady-state shock-wave position $x=x_{s 0}$.

Analytical solutions can be derived in special cases. For symmetric parabolic walls, as noted earlier, $f(0)-f(x)$ $=1 / 2(\gamma+1) u_{l \infty}^{2}\left(x / x_{l}\right)^{2}$ and $x_{l}=-x_{0}$. If the walls undergo a symmetric simple harmonic pitching oscillation with constant pressure downstream, $G(x, t)=\left(x-x_{c}\right) \beta_{o} e^{i t}$ and $G_{d}(t)=0$. The leading edges are now wedge shaped rather than cusped, and so a different entrance solution is needed. The perturbation potential is $\Theta\left(\epsilon^{2}\right)$ and the largest term satisfies Laplace's equation in the coordinates $\hat{x}, y$ with the boundary condition $v \sim$ const at the interior walls. Again, with the help of the mapping [Eq. (29)], the solution is found to be

$(K \epsilon)^{1 / 2}\left(u-u_{\infty}\right)-i v=\epsilon^{2} \frac{\gamma+1}{\pi x_{I}} u_{I \infty}^{2}\left\{\ln \left(1-\frac{1}{\zeta^{2}}\right)+\frac{1}{\zeta^{2}-1}\right\}+\ldots$

The form of the solution as $z^{*} \rightarrow \pm i$ shows that there is an outward flow around the edges. 
In the same case, for the flow upstream of the shock wave, the solution of the linear equation (57) for $h_{2 x}$ is

$$
\begin{aligned}
h_{2 x} & =\left(\frac{u_{l \infty}}{x_{1}}\right)^{2}\left\{\left(\frac{1}{2}-\frac{\gamma}{3}\right) x^{2}-\frac{\gamma+l}{6}\right\} \\
& +\frac{\beta_{0} x_{1} e^{i(t-x)}}{(\gamma+1)\left|u_{l \infty}\right|\left(1+\nu^{2}\right)^{3 / 2}}
\end{aligned}
$$

where $\nu=\tan \chi=2 k(\gamma+1)^{-1} x_{1} /\left|u_{I \infty}\right|$. Outside the channel the speed $|u-a|$ at which small disturbances travel upstream becomes $u_{\infty}-a_{\infty}-1 / 2(\gamma+1) \epsilon u_{1 \infty}+\ldots$. The parameter $\nu$ is a reduced frequency based on this velocity, the frequency $k \epsilon$, and the length $x_{1}$. The phase lag angle $\chi$ increases from zero to $\pi / 2$ as $\nu$ increases from zero to infinity, and $\chi$ increases as the magnitude of the wall curvature $(\gamma+1) u_{1 \infty}^{2} / x_{I}^{2}$ decreases. The solution of the differential equation $(60)$ for $\lambda(t)$ is easily found to be the same as the second term in $h_{2 x}$, as is required for proper matching.

Downstream of the shock wave the solution to Eq. (57) requires additional terms from the solution to the homogeneous equation so that the downstream boundary condition can be satisfied. The result is

$$
\begin{aligned}
h_{2 x} & =\left(\frac{u_{1 \infty}}{x_{I}}\right)^{2}\left\{\left(\frac{1}{2}-\frac{\gamma}{3}\right) x^{2}-\frac{\gamma+1}{6}\right\}-\frac{c_{2 d}^{(s)} x_{I}}{(\gamma+1)\left|u_{I \infty}\right| x} \\
& -\left\{1-\frac{x_{1}}{x} \exp \left(i \nu \ln \frac{x}{x_{1}}\right)\right\} \frac{\beta_{0} x_{1} e^{i(t+x)}}{(\gamma+I)\left|u_{1 \infty}\right|\left(1+\nu^{2}\right)^{1 / 2}}
\end{aligned}
$$

The time-dependent part of the solution to the homogeneous equation has constant phase $t+\chi+\nu \ln \left(x / x_{1}\right)$ along the characteristics $\mathrm{d} x / \mathrm{dt}=-x / \nu$ and hence describes disturbances which travel upstream at this speed from the channel exit. Again the particular solution gives the direct response to the motion of the walls. The in-phase part has a different sign than in the upstream solution [Eq. (63)] because positive $G_{x}(x, t)$ now implies a velocity decrease instead of an increase, as was true in the supersonic flow ahead of the shock wave; this is more clear in the limit as $\nu \rightarrow 0$. The out-of-phase part retains the same sign as in the upstream solution, since it occurs as a consequence of the $\phi_{x t}$ term, which has a damping effect on the fluid motion and introduces a phase lag, as in the forced response of a damped linear oscillator. The motion of the shock wave now can be determined from Eq. (61), with $\left(h_{2 x}\right)_{u}$ and $\left(h_{2 x}\right)_{d}$ found by evaluating Eqs. (63) and (64) at $x=x_{50}$. The constant value $x_{50}$ is found by setting the time average of $\mathrm{d} x_{s I} / \mathrm{d} t$ equal to zero, so that $c_{2 d}^{(s)}$ has the same value as in the steady-flow case. The results are

$$
\begin{gathered}
c_{2 d}^{(s)}=-\frac{2 \gamma(\gamma+1)}{3}\left(\frac{\left|u_{l \infty}\right| x_{s 0}}{x_{1}}\right)^{3} \\
k \frac{\mathrm{d} x_{s I}}{\mathrm{~d} t}=\frac{\beta_{0} x_{1} e^{i t}}{4\left|u_{1 \infty}\right|\left(1+v^{2}\right)^{1 / 2}}\left\{-2 i \sin \chi+\frac{x_{1}}{x_{s 0}} \exp \left[i\left(\chi-\nu \ln \frac{x_{I}}{x_{s 0}}\right)\right]\right\}
\end{gathered}
$$

The first term contains the effects of the upstream and downstream changes in flow velocity which result directly from the wall motion, and is $180 \mathrm{deg}$ out of phase with the velocity $i \beta_{0}\left(x-x_{c}\right) e^{i t}$ of the wall motion. The second term in $\mathrm{d} x_{s I} / \mathrm{d} t$ represents the effect of flow disturbances which arise because the back pressure is prescribed and which travel upstream at a speed $u-a$ from the channel exit. The phase lag $\nu \ln \left(x_{t} / x_{s \theta}\right)$ is equal to the time $\Delta t$ required for a disturbance to travel upstream from the exit $x=x_{1}$ to the shock wave at $x=x_{s 0}+\ldots$ at a speed $u-a=-1 / 2 \epsilon(\gamma+1)\left|u_{l \infty}\right| x / x_{I}+\ldots$.
The simple form of the solutions for this special case permits a comparison of results for $\tau^{-1}=\mathcal{O}\left(\epsilon^{2}\right)$ and $\tau^{-1}$ $=\mathcal{O}(\epsilon)$, both for $\alpha=\epsilon^{3}$. The general principle is that the solutions for $\tau^{-1}=\mathcal{Q}\left(\epsilon^{2}\right)$ evaluated as $\tau \epsilon^{2} \rightarrow 0$ should agree with the solutions for $\tau^{-1}=\mathcal{O}(\epsilon)$ evaluated as $\tau \epsilon \rightarrow \infty$. The present solutions can be investigated as $\tau \epsilon \rightarrow \infty$ by taking the limit as $k \rightarrow 0$. It can be shown in this way that the shock-wave velocity and the flow velocities do satisfy the required condition, and that the solutions for the flow velocities when $\tau^{-1}$ $=\mathcal{O}\left(\epsilon^{2}\right)$ are in fact limiting cases of the corresponding solutions found when $\tau^{-1}=\mathcal{O}(\epsilon)$.

\section{Oscillations of Larger Amplitude}

When $\alpha=\epsilon^{2}$, the amplitude of the wall oscillations is of the same order as the wall thickness. If again $\tau^{-1}=k \epsilon$, the time dependence in the expansion equation (14) first appears in $\phi_{l}$, so that $\phi_{1}=\phi_{1}(x, t)$. The solution for $\phi_{2}$ is similar in form to the solution (19), but the coefficient of $y^{2}$ is now $1 / 2\left(-f^{\prime}+G_{x}\right)$, and $h_{2}(x, t)$ now satisfies a more complicated differential equation which contains the first approximation to the change $H-H_{\infty}$ in total enthalpy. It follows from Eq. (7) for $\mathrm{D} H / \mathrm{D} t$ that upstream of the shock wave $H-H_{\infty}=$ $-k \epsilon \phi_{i t}+\ldots$, where the term of order $\epsilon$ in $\phi$ has been taken equal to zero in the undisturbed flow. Downstream of the shock wave $H$ contains an additional term of this order, as found from Eq. (11).

The procedures used in deriving Eq. (18) for $u_{I}=\phi_{l x}$ now give a differential equation

$$
2 k \phi_{1 x t}+(\gamma+1) \phi_{1 x} \phi_{1 x x}=-f^{\prime}+G_{x}
$$

For the subsonic flow downstream of the shock wave, the characteristics $\mathrm{d} x / \mathrm{d} t=(\gamma+1) u_{l} /(2 k)$ have negative slope in the $x, t$ plane; small disturbances propagate in the upstream direction, and the initial condition for Eq. (66) is given at $x=x_{1}$. For the flow upstream of the shock wave, the characteristics have positive slope if $u_{1}>0$ and negative slopes if $u_{1}<0$, so that small disturbances always propagate away from the sonic line $x=x_{*}(t)+\ldots$ where $u_{l}=0$, and the initial condition for Eq. (66) should be a condition at the sonic line. The shock-wave displacement $x=x_{s 0}(t)$ has amplitude $\Theta(1)$, with $x_{s 0}$ found from

$$
\frac{4 k}{\gamma+1} \frac{\mathrm{d} x_{s 0}}{\mathrm{~d} t}=u_{I d}+u_{I u}
$$

where $u_{l d}$ and $u_{I u}$ are obtained from the solutions for $u_{l}(x, t)$.

Equation (66) can be rewritten in terms of a coordinate $s$ measured along characteristics (a convenient choice is $s=t$ ) and a coordinate $r$ which is constant along each characteristic. In this way a pair of first-order equations is obtained for $x(r, s)$ and $u_{l}(r, s)$. For the example discussed above, with parabolic walls undergoing simple harmonic pitching oscillations, these equations are linear, and the complex form $G=\beta_{0}\left(x-x_{c}\right) e^{i t}$ again can be used. The general solution then is found easily, and contains two arbitrary functions of $r$. Upstream of the shock wave the requirement that $u_{1}$ must remain finite at the throat leads to the result

$$
\begin{gathered}
\frac{u_{l}}{\left|u_{l \infty}\right|}=\frac{x-x_{*}(\mathrm{t})}{x_{I}} \\
x_{*}(t)=-\frac{\beta_{0} x_{I}^{2}(1-i \nu)}{(\gamma+1) u_{l \infty}^{2}\left(1+\nu^{2}\right)} e^{i t}
\end{gathered}
$$

Derivation of a local solution for $x-x_{*}=\mathcal{O}\left(\epsilon^{1 / 2}\right)$ verifies that the solution [Eq. (68)] does remain correct near the sonic line. The instantaneous cross-sectional area of the channel has a minimum at the location $x=x_{\min }$ where the right-hand side 
of Eq. (66) is zero. By comparison with Eq. (68), it is seen that $x_{*}=x_{\min } /(1+i \nu)$, and so the sonic line no longer occurs at the cross section having minimum area. Since $0<\arg (1+i v)<\pi / 2$ for $0<\nu<\infty$, the displacement of the sonic line lags the wall motion by a phase angle between 0 and $\pi / 2$; the amplitude in Eq. (68) decreases as the frequency increases, with $x_{*} \rightarrow 0$ as $\nu \rightarrow \infty$.

For the flow downstream of the shock wave, the unknown functions of $r$ which appear in $x(r, s)$ and $u_{l}(r, s)$ are determined by conditions imposed at $x=x_{1}$. To find the shockwave position in the form $r=R(s)$, after the real part has been taken for $x(r, s)$ and $u_{l}(r, s)$, it is necessary to solve a nonlinear first-order ordinary differential equation for $R(s)$. This equation is derived in Ref. 7; no numerical work has been carried out for this case.

\section{Concluding Remarks}

The present results extend the solutions of Ref. 3 to include effects of wall oscillations as well as oscillations in back pressure. A calculation of the flow near the channel entrance allows application of the results to inlet flows. Additional details have been obtained near the throat, and a solution has been given for the velocity of a shock wave which moves upstream through the subsonic flow ahead of the throat. Other extensions include the study of flow problems corresponding to different orders of magnitude for the frequency and amplitude of the impressed disturbances. In one case large-amplitude shock-wave motion again results, and the sonic line need not lie close to the instantaneous position of the minimum channel cross-sectional area.

\section{Acknowledgment}

This work was supported in part by Applied Analysis, Inc., through Contract N00019-80-C-0587 with Naval Air Systems Command; this support is gratefully acknowledged.

\section{References}

${ }^{1}$ Richey, G. K. and Adamson, T. C. Jr., "Analysis of Unsteady Transonic Channel Flow with Shock Waves," AIAA Journal, Vol. 14, Aug. 1976, pp. 1054-1061.
${ }^{2}$ Chan, J. S-K. and Adamson, T. C. Jr., "Unsteady Transonic Flows with Shock Waves in an Asymmetric Channel," AIAA Journal, Vol. 16, April 1978, pp. 377-384.

${ }^{3}$ Adamson, T. C. Jr., Messiter, A. F., and Liou, M. S., "Large Amplitude Shock-Wave Motion in Two-Dimensional Transonic Channel Flow," AIAA Journal, Vol. 16, Dec. 1978, pp. 1240-1247.

${ }^{4}$ Adamson, T. C. Jr. and Liou, M. S., "Unsteady Motion of Shock Waves in Two Dimensional Transonic Channel Flows," University of Michigan, Ann Arbor, Mi., Rept. UM 014534-F, June 1977.

${ }^{5}$ Messiter, A. F. and Adamson, T. C. Jr., "Asymptotic Solutions for Nonsteady Transonic Channel Flows," Symposium Transsonicum $I I$, edited by K. Oswatitsch and D. Rues, Springer-Verlag, New York, 1976, pp. 41-48.

${ }^{6}$ Messiter, A. F. and Adamson, T. C. Jr., "Transonic SmaillDisturbance Theory for Lightly Loaded Cascades," AIAA Journal, Vol. 19, Aug. 1981, pp. 1047-1054.

${ }^{7}$ Adamson, T. C. Jr. and Messiter, A. F., "Unsteady Transonic Flows in Two-Dimensional Channels with Oscillating Boundaries," Applied Analysis, Inc., Ann Arbor, Mi., Rept. AAI-0587-FT, 1981.

${ }^{8}$ Messiter, A. F. and Adamson, T. C. Jr., "On the Flow near a Weak Shock Wave Downstream of a Nozzle Throat," Journal of Fluid Mechanics, Vol. 69, 1975, pp. 97-108.

${ }^{9}$ Platzer, M. F., "Unsteady Flow in Turbomachines-A Review of Current Developments," Unsteady Aerodynamics, AGARD-CP-227, 1977, pp. 33-1-33-28.

${ }^{10}$ Meier, G. E. A., "Shock Induced Flow Oscillations," Flow Separation, AGARD-CP-168, 1975, pp. 29-1-29-9.

${ }^{11}$ Sajben, M., Kroutil, J. C., and Chen, C. P., "Unsteady Transonic Flow in a Two Dimensional Diffuser," Unsteady Aerodynamics, AGARD-CP-277, 1977, pp. 13-1-13-14.

${ }^{12}$ Liou, M. S. and Sajben, M., "Analysis of Unsteady Viscous Channel Flow with a Shock Wave in a Two-Dimensional Channel," AIAA Paper 80-0195, 1980.

${ }^{13}$ Liou, M. S., "Analysis of Viscous-Inviscid Interactions in Transonic Internal Flows with a Shock Wave and Mass Transfer," AIAA Paper 81-0004, 1981.

${ }^{14}$ Liou, M. S. and Coakley, T. J., "Numerical Simulations of Unsteady Transonic Flow in Diffusers," AIAA Paper 82-1000, 1982.

${ }^{15}$ Sajben, M., Bogar, T. J., and Kroutil, J. C., "Forced Oscillation Experiments in Supercritical Diffuser Flows with Application to Ramjet Instabilities," AIAA Paper 81-1487, 1981.

${ }^{16}$ Adamson, T. C. Jr., "Unsteady Transonic Flows in TwoDimensional Channels,"’ Journal of Fluid Mechanics, Vol. 52, 1972, pp. 437-449. 